\title{
A New Composite Electrode Applied for Studying the Electrochemistry of Insoluble Particles: $\alpha-\mathrm{HgS}$
}

\author{
Minjun Yang', Richard G. Compton ${ }^{1}\left(\Xi_{\circledast}\right)$ \\ ${ }^{1}$ Department of Chemistry, Physical and Theoretical Chemistry Laboratory, University of Oxford, South Parks \\ Road, Oxford OX1 3QZ, United Kingdom
}

\begin{abstract}
The redox chemistry of solid $\alpha$-HgS particles is revealed using a carbon/PVDF composite containing $\alpha$-HgS, carbon black, polyvinylidene fluoride (PVDF). The electrochemical behaviour of the carbon/PVDF composite is first characterised with three water insoluble organic solids. Then the reduction of solid $\alpha$-HgS particles is investigated and found to occur at a high negative potential, $1.82 \mathrm{~V}$ vs saturated mercury sulphate reference electrode, to form metallic mercury and sulphide ions. The subsequent oxidation of metallic mercury and sulphide occurs at $+0.24 \mathrm{~V}$ and $-0.49 \mathrm{~V}$ vs MSE respectively.
\end{abstract}

\section{KEYWORDS}

Mercury (II) sulphide; $\alpha$-HgS; Cinnabar; Carbon black; Polyvinylidene fluoride; Solid state electrochemistry

Address correspondence to Richard G. Compton, Richard.compton@ chem.ox.ac.uk 


\section{Introduction}

Natural occurring forms of mercury are found commonly as minerals of which the most important are red cinnabar and black metacinnabar. The red cinnabar, $\alpha-\mathrm{HgS}$, is more abundant than the black metacinnabar ( $\beta-\mathrm{HgS}$ ) and it is commonly found near volcanos and has been used as pigments and in cosmetics since ancient times. Metallic mercury can be extracted by heating solid $\alpha-\mathrm{HgS}$ with a flux of air followed by vapour condensation. Mercury and mercury salts are commonly known for their high toxicity. ${ }^{[1]}$ Disposal of untreated mercury waste waters into the aquatic environment forms methylmercury by bacteria methylation, ${ }^{[2]}$ a neurotoxin responsible for Minamata disease. ${ }^{[3]}$ Despite the notorious toxicity of mercury compounds, some traditional Chinese medicines retain the use of $\alpha-\mathrm{HgS}$ as part of their recipes. A commercial Chinese medicine, An-Gong-Niu-Huang Wan, is currently banned for sales in USA and Japan due to the cinnabar and realgar $\left(\alpha-A s_{4} S_{4}\right)$ content. ${ }^{[4]}$ At room temperature, $\alpha-\mathrm{HgS}$ is thermodynamically more stable than $\beta$-HgS. ${ }^{[5]}$ However, it is $\beta$-HgS that usually precipitates from the solution phase. ${ }^{[5 c, 6]}$ The transition temperature of cinnabarmetacinnabar for stoichiometric mercury sulphide at 1 atmospheric pressure is reported to be $643 \mathrm{~K} \cdot{ }^{[5 b, 6 a, 7]}$ Both $\alpha-\mathrm{HgS}$ and $\beta-\mathrm{HgS}$ are extremely insoluble in aqueous solution, the logarithm of the corresponding solubility products were reported around -53 and -52 at $298 \mathrm{~K}$ respectively. ${ }^{[5 c, 8]}$ Perhaps due to its insolubility, the toxicity of mercury sulphides are relatively low compared to other forms of mercury. ${ }^{[4]}$

The electrochemistry are well-developed for dissolved organic and inorganic compounds. ${ }^{[9]}$ In contrast, electrochemical analysis on solids was faced with many challenges implicit in a quote from Scholz: "...[the] problems involved are severe and success was rather rare...". ${ }^{10]}$ In 1958, Ralph N. Adams developed a carbon paste electrode (CPE) to study the electrochemistry of insoluble compounds. ${ }^{[11]}$ Carbon paste electrodes were made by packing a mixture of graphite, insoluble compounds and organic oil into a hollow teflon tube. This method was developed extensively by the electrochemists in the USSR ${ }^{[12]}$ as reviewed by Scholz. ${ }^{[13]}$ More recent and comprehensive reviews on the applications and developments of carbon paste electrode were reported by Švancara et.al. ${ }^{[14]}$ In the late twentieth century, other electrochemical methods such as abrasive attachment were used for the electrochemical study of insoluble solids. ${ }^{[13 a]}$ In 1991, a reductive differential pulse voltammogram of $\alpha-\mathrm{HgS}$ was reported by Scholz et.al with a large reductive peak seen at $-1.5 \mathrm{~V}$ vs $\mathrm{SCE} ;{ }^{[15]}$ solid $\alpha-\mathrm{HgS}$ was "mechanically" transferred to a paraffin impregnated graphite electrode. ${ }^{[10]}$ Generally, the redox products of solid particles are typically water soluble. Depending on the electrochemical system of the solid of interest, the products may diffuse into the bulk solution or 
remain on the electrode. Previously, polymer films have been used to immobilise solid particles on electrodes and sample "recuperation" was seen after the electrochemical measurements. ${ }^{[16]}$

Herein, a new composite containing carbon black, polyvinylidene fluoride (PVDF), N-Methyl-2pyrrolidone (NMP) and an insoluble solid of interest was dropcasted onto a glassy carbon electrode and subsequently dried to form a carbon based composite electrode. The PVDF binds the solid of interest whilst the carbon black confers electronic conductivity. At the same time the composite is sufficiently porous for the solid under study to 'see' electrolyte solution. Together, the layers of carbon and PVDF hinder the diffusion of the redox products into the solution, even when products are normally water soluble. The characteristics of this composite are first compared to other previously developed methodologies using three well-studied water insoluble organic compounds; namely 9,10-anthraquinone, oil blue $\mathrm{N}$ dye and indigo. Having characterised the intrinsic properties of this carbon/PVDF composite, the focus then turns to the electrochemistry of $\alpha-\mathrm{HgS}$. The use of this carbon/PVDF composite reveals a cyclic voltammogram of solid $\alpha-\mathrm{HgS}$ for the first time and shows the subsequent oxidation of sulphide and anodic stripping of metallic mercury.

\section{Experimental}

\section{Chemicals}

All chemicals used were of analytical grade and were purchased from Sigma-Aldrich except carbon black $^{[17]}$ (diameter $27 \pm 10 \mathrm{~nm}$, Monarch 430, Cabot Performance). The Polyvinylidene fluoride (PVDF), supplied by Sigma-Aldrich, has an average molecular weight of $5.34 \times 10^{5}$ by GPC. These chemicals were used without further purification. Aqueous solutions were made using ultrapure water (Millipore, resistivity not less than $18.2 \mathrm{M} \Omega \mathrm{cm}$ at $25^{\circ} \mathrm{C}$ ). Nitrogen gas $(99.998 \%$, BOC Gases plc, Guildford UK) was used to de-oxygenate the electrolytic solutions at a sufficient intensity and for a sufficient period of time to ensure no measurable oxygen signal is observed in the voltammogram except where discussed below.

\section{Carbon/PVDF composite modified electrodes and electrochemical equipment}

The carbon/PVDF composite contains a mixture of insoluble material, carbon black and PVDF (dissolved in NMP, $25 \mathrm{mg} / \mathrm{ml}$ ) in weight ratio of 20:60:20 unless otherwise specified. This weight ratio was selected for a balance of electrochemical signal for the solid of interest whilst maintaining electrical contact with the carbon black and enough PVDF to maintain a strong physical binding. The carbon/PVDF composites were stirred overnight and $2 \mu \mathrm{l}$ was dropcasted onto a glassy carbon 
working electrode (radius $=1.50 \mathrm{~mm}, \mathrm{CH}$ instruments, $\mathrm{TX}, \mathrm{USA}$ ). The modified working electrode was subsequently dried overnight at $70^{\circ} \mathrm{C}$ to evaporate off the NMP solvent.

A saturated mercury sulphate electrode (ALS, Japan) was used as the reference electrode and the three electrode cell configuration was completed using a carbon rod counter electrode. The electrochemical experiments were conducted with a computer controlled Autolab potentiostat 30 (Metrohm Auotlab B.V., Utecht, The Netherlands) using NOVA 2.0 with the "alpha value" set to 0.3, which controls the point of current sampling in the staircase ramp. ${ }^{[18]}$ The carbon/PVDF composite modified GC electrode was polished after each experiment with 3, 1, $0.1 \mu \mathrm{m}$ diamond spray (Kemet, UK) in descending order of size. The diamond spray residue was then removed from the electrode surface by rinsing with excess ultrapure water and subsequently dried under a nitrogen flow.

\section{Buffer solutions}

The $\mathrm{pH}$ buffer solutions used contained a diversity of $0.1 \mathrm{M} \mathrm{KNO}_{3}$ based solutions, as shown in Table 1. The precise $\mathrm{pH}$ was adjusted to the indicated value using small amounts of $1 \mathrm{M} \mathrm{HCl} / \mathrm{NaOH}$. The $\mathrm{pH}$ measurement was performed with a pH 213 microprocessor pH meter (HANNA Instruments, Leighton Buzzard, UK) and a pH electrode (HI 1131, HANNA Instruments), which was calibrated prior to use.

Table 1: Composition of the $\mathrm{pH}$ buffered solutions in $0.1 \mathrm{M} \mathrm{KNO} \mathrm{K}_{3}$ electrolyte.

\begin{tabular}{|c|c|c|c|c|c|c|}
\hline & Solution com & sition in $0.1 \mathrm{M} \mathrm{KNC}$ & $/ \mathrm{mL}$ & & & \\
\hline $\mathrm{pH}$ & $\begin{array}{l}0.1 \mathrm{M} \text { citric } \\
\text { acid }\end{array}$ & $\begin{array}{l}0.1 \mathrm{M} \text { potassium } \\
\text { phosphate } \\
\text { dibasic }\end{array}$ & $\begin{array}{l}0.1 \mathrm{M} \text { potassium } \\
\text { phosphate } \\
\text { monobasic }\end{array}$ & $\begin{array}{l}0.1 \mathrm{M} \text { sodium } \\
\text { carbonate }\end{array}$ & $\begin{array}{l}0.1 \mathrm{M} \text { sodium } \\
\text { bicarbonate } \\
\text { solution }\end{array}$ & $\begin{array}{l}0.1 \mathrm{M} \text { sodium } \\
\text { hydroxide }\end{array}$ \\
\hline $\begin{array}{l}5.0 \\
7.0 \\
9.0 \\
11.0 \\
13.0\end{array}$ & 24.3 & $\begin{array}{l}51.4 \\
61.0\end{array}$ & 39.0 & $\begin{array}{l}4.0 \\
42.5\end{array}$ & $\begin{array}{l}46.0 \\
7.5\end{array}$ & 100.0 \\
\hline
\end{tabular}

\section{Scanning electron microscopy}

Scanning electron microscope images were taken on Zeiss Sigma 300 FEG-SEM at an accelerating voltage of $10.0 \mathrm{KV}, 15 \mu \mathrm{m}$ aperture and 3610X magnification. The carbon/PVDF composites were stirred overnight. The carbon fibre stub was polished with aqueous slurries of $1,0.3$ and $0.05 \mu \mathrm{m}$ alumina in decreasing order of size, follow by 30 minutes of sonication and immersed in aqua regia overnight before rinse and dry. $2 \mu \mathrm{L}$ of the carbon/PVDF composite was then dropcasted onto the carbon fibre stub and dried overnight at $70^{\circ} \mathrm{C}$. 


\section{Results and discussion}

The electrochemical behaviour of the carbon/PVDF composite is first studied with three well-known water insoluble organic compounds; namely 9,10-anthraquinone, oil blue $\mathrm{N}$ dye and indigo. The mechanistic pathways and electrochemical reactions of those organic compounds are well known but are studied to characterise the behaviour of the composite to validation. Subsequent attention is focused on the electrochemistry of $\alpha$-HgS.

\section{9,10-anthraquinone}<smiles>O=C1c2ccccc2C(=O)c2ccccc21</smiles>

$\mathrm{AQ}$<smiles>Oc1c2ccccc2c(O)c2ccccc12</smiles>

$\mathrm{AQH}_{2}$

Figure 1. Chemical structure of 9,10-anthraquinone(AQ) and the electrochemical reactions.

The reduction process of quinones are generally two-electron transfer in different media to form the corresponding hydroquinone or quinone dianion. ${ }^{[19]}$ In organic aprotic solvents, 9,10-anthraquinone (AQ) and similar derivatives undergoes two successive one-electron reductions to form the corresponding semiquinone $A Q^{-}$and the dianion $\mathrm{AQ}^{2-}$; two separate waves can be seen on the reductive sweep. ${ }^{[20]}$ Since $A Q$ is insoluble in water, electrochemical studies have usually been conducted with either surface bound $A Q$ or similar derivatives that are water soluble. ${ }^{[21]}$ In acidic buffered aqueous solutions, the overall reduction pathway is a stepwise two-electron and twoproton via a "scheme-of-squares" ${ }^{[22]}$ to form the corresponding hydroquinone. ${ }^{[21,23]}$ In alkaline buffered aqueous solutions, the reduction process is two stepwise electron transfer reactions to form the corresponding quinone dianion. ${ }^{[21]}$ Note, only one apparent wave was seen on the reduction in buffered aqueous solutions across all $\mathrm{pH}$ values, ${ }^{[24]}$ this is ascribed to the hydrogen bonding stabilisation leading to "potential inversion" at the quinone/semiquinone and semiquinone/hydroquinone couple. ${ }^{[25]}$

The cyclic voltammogram recorded on an $A Q$ carbon/PVDF composite working electrode in $\mathrm{pH} 13$ $\mathrm{NaOH}$ buffered solution is depicted in Figure 2. On the first cycle, a high negative over-potential was 
required to initiate the redox chemistry of solid $A Q$. On subsequent cycles, redox signals corresponding to the two-electron transfer reaction were seen with a formal potential around -1.19 V vs MSE. ${ }^{[26]}$ This initiation process in the first cycle is familiar as a "break-in" reaction in solid state electrochemistry. ${ }^{[27]}$ In solution phase electrochemistry, charge neutrality can be rapidly achieved by diffusion of nearby electrolyte upon electron transfer. In solid state electrochemistry however, the electrolyte typically cannot diffuse freely into the solid crystalline and over-potential is required to 'break' the structural integrity. The break-in reaction was only observed on the first cycle. It is likely that upon the initial reduction, the formed $A^{2-}$ species diffuses within the composite and on the reverse sweep, is oxidised to form smaller sized $A Q$ crystalline. The peak current decreases upon consecutive cycles, indicates some loss of material from the electrode during each cycle. This is likely due to the escape of the water soluble product $\mathrm{AQ}^{2-}$ into the bulk solution by diffusion. Note, a large IR drop was observed in the cyclic voltammogram of $A Q$, which results in the large scale of the peak to peak separation.

For the voltammetry of non-diffusing species, the peak current scales linearly with scan rate with a intercept at zero. ${ }^{[9 a, 28]}$ In the case of $A Q$ carbon/PVDF composite, the break-in reaction takes place on the first cycle and therefore the relationship of peak current and scan rate were investigated on the $2^{\text {nd }}$ cycle, shown in Figure 3 . The inlay of Figure 3 shows a plot of peak current against scan rate. As can be seen, the relationship of peak current and scan rate deviates from linearity at high scan rates. This can be in part ascribed to the reduced time available for the break-in reaction to occur completely on the first cycle. Therefore, less 'activated' AQ are available for reduction on the second cycle which leads to a decrease in peak current.

In comparison to the voltammogram of solid phase AQ reported by Kuwana and French using a $\mathrm{CPE}^{\left[{ }^{[26]}\right.}$ the break-in reaction was observed using the carbon/PVDF composite but not with a CPE where the $A Q$ is likely dissolved in the oil used as binder. The carbon/PVDF composite is therefore a complementary approach to the CPE for a thorough electrochemical analysis of solid such as AQ.

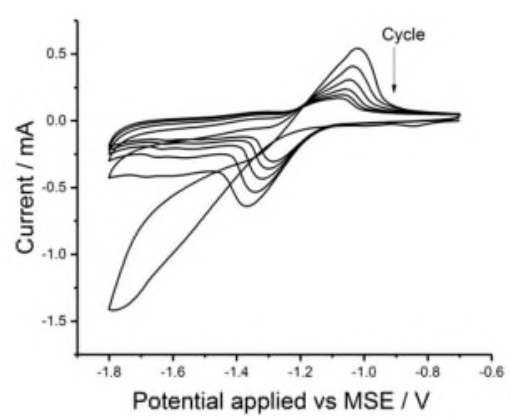


Figure 2. Cyclic voltammogram recorded on an 9,10-anthraquinone carbon/PVDF composite modified electrode. The first 6 cycles are shown and recorded consecutively in degassed solution containing $0.10 \mathrm{M} \mathrm{KNO}_{3}$ electrolyte at a scan rate of $30 \mathrm{mVs}^{-1}$. The composite contains a mixture of 9,10-anthraquinone, carbon black and PVDF in weight ratio of 20:60:20.

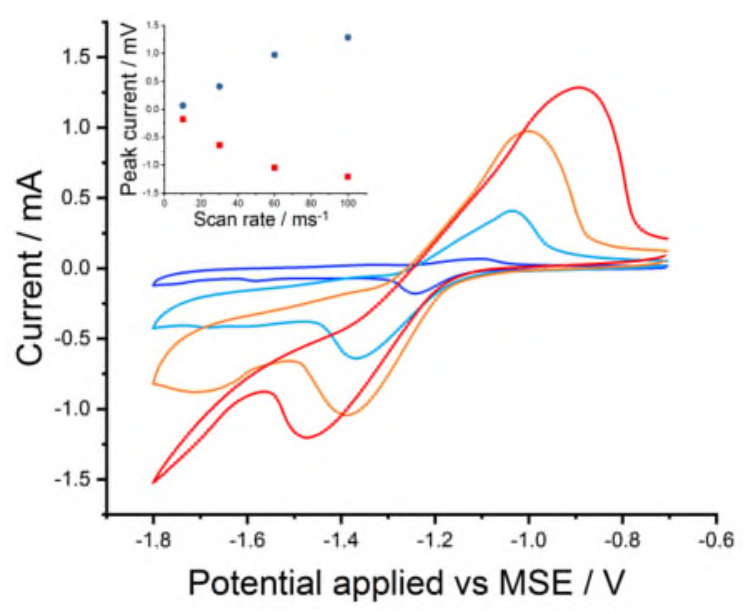

Figure 3. The $2^{\text {nd }}$ cycle recorded on an 9,10-anthraquinone carbon/PVDF composite modified electrode at different scan rates. The solution is degassed and contains $0.10 \mathrm{M} \mathrm{KNO}_{3}$ electrolyte. The scan rates of 10,30,60 and 100 $\mathrm{mVs}^{-1}$ are displayed with dark blue, blue, orange and red line. Inlay, peak current verses scan rate. The composite contains a mixture of 9,10-anthraquinone, carbon black and PVDF in weight ratio of 20:60:20.

The key behaviour of the composite can be deduced from the AQ carbon/PVDF composite; the charged molecules formed from the redox reaction, such as $\mathrm{AQ}^{2-}$, can diffuse out of the composite but only slowly in the voltammertic time scale. This was evidenced by the relatively weak decrease in peak current upon consecutive cycles in contrast to "abrasive voltammetry". The focus in next section turns to the electrochemistry of oil blue $\mathrm{N}$ dye in carbon/PVDF composite.

\section{Oil blue $\mathrm{N}$ dye}
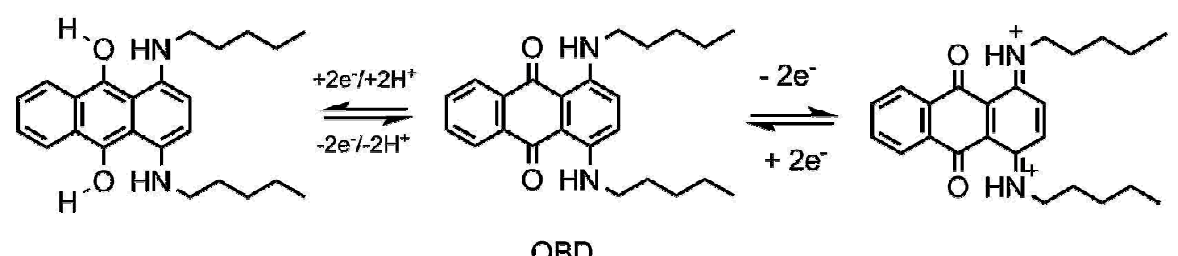

Figure 4. Chemical structure of oil blue $N$ dye $(O B D)$ and the electrochemical reactions.

The redox chemistry of oil blue $N$ dye (OBD, Figure 4) is known to be bifunctional, such that both reduction and oxidation can occur. ${ }^{[29]}$ The processes in water are the two-electron reduction of the quinone function group and the two-electron oxidation on the phenylenediamine group, as reported previously. ${ }^{[29]}$ The cyclic voltammograms obtained for the reduction of oil blue $\mathrm{N}$ dye carbon/PVDF composite in aqueous solution are shown in Figure 5. Reductive signal were observed at potentials of $-0.97 \mathrm{~V}$ and $-1.11 \mathrm{~V}$ vs MSE respectively, the latter is attributed to the two-electron reduction of 
the quinone group. ${ }^{[21,30]}$ The reduction peak at $-0.97 \mathrm{~V}$ vs MSE is ascribed to the reduction of oxygen, as it is also observed in the control experiment measured using a 'blank' carbon/PVDF composite unloaded with OBD. Note that the oxygen is present in the composite despite degassing the solution to the extent that would not give voltammertic signal on a non-porous electrode.

The oxidation of OBD in the carbon/PVDF composite is shown in the inlay of Figure 5 . The first cycle (orange line) shows little redox activity in comparison to the second cycle (red line), likely due to the occurrence of a "break-in" reaction as observed with the reduction of 9,10-anthraquinone. The second cycle of the oxidation of OBD shows oxidative peaks at +0.12 and $+0.40 \mathrm{~V}$ vs MSE, the peak potentials are in agreement with previous report by direct dropcast of OBD on a glassy carbon electrode. ${ }^{[29]}$ A back wave can be seen on the oxidation of OBD with the use of carbon/PVDF composite but not when directly dropcast onto an electrode surface. ${ }^{[29]}$ The oxidised species of OBD will likely be water soluble as it bears a positive charge. Therefore, it likely completely diffuses and escapes into the bulk solution from a direct dropcast onto an electrode surface. By using the carbon/PVDF composite, the diffusion oxidised OBD species is hindered by the layers of carbon held together using PVDF and hence a back wave is seen on the reverse sweep.

The redox potentials of OBD obtained using the carbon/PVDF composite are consistent with the direct $\mathrm{OBD}$ dropcast measurement. Moreover, since the formed products do not completely diffuse out of the carbon/PVDF composite layers within the experimental time scale, additional electrochemical information can be obtained for the redox couple. The presence of oxygen in the carbon/PVDF composite may distort the voltammetric signal of other solids of interest; this can be overcome by increasing the weight ratio of the solid of interest or preparing the carbon/PVDF composite electrode and conducting the experiment inside a glove box, under a nitrogen atmosphere. The next section will discuss the behaviour of the carbon/PVDF composite in $\mathrm{pH}$ buffered solutions.

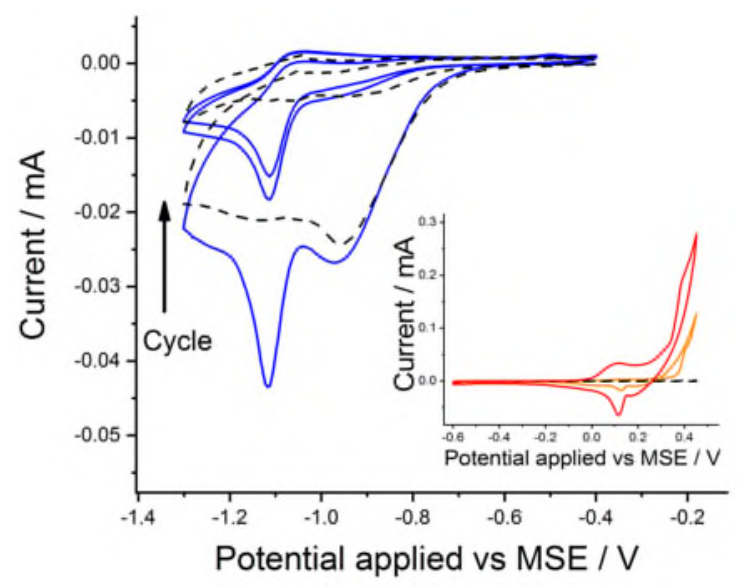


Figure 5. Cyclic voltammograms of an oil blue $N$ dye carbon/PVDF composite modified electrode. The voltammogram swept reductively from - $0.40 \mathrm{~V}$ and returns at $-1.30 \mathrm{~V}$ vs MSE. Inlay, voltammogram swept positively from -0.60 V to +0.45 V vs MSE at scan rate of $10 \mathrm{mVs}{ }^{-1}$. Solid lines - composite of oil blue $N$ dye, carbon black and PVDF in weight ratio of 20:60:20. Dotted line - control experiment measured on a blank carbon/PVDF composite (carbon black and PVDF, weight ratio of 60:20) modified electrode. The measurements was conducted in a degassed solution contains $0.1 \mathrm{M} \mathrm{KNO}_{3}$ electrolyte.

Indigo

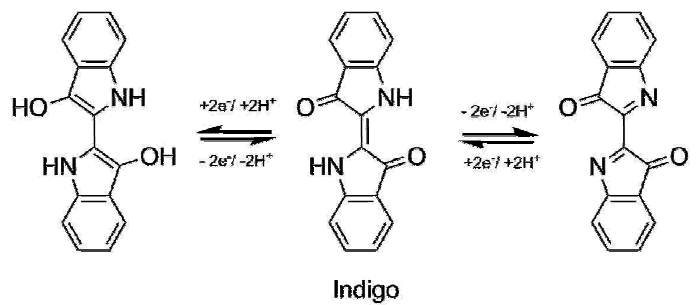

Figure 6. Chemical structure of indigo and the electrochemical reactions in the presence of protons.

The natural occurring dye indigo is used for textile dyeing, most commonly used for denim cloth, due to its vibrancy and distinctive deep blue hue. ${ }^{[31]}$ To meet the demand of the fashion in denims, 50,000 tonnes of indigo have been produced synthetically per year since $2011 .{ }^{[32]}$ As indigo is water insoluble, it cannot penetrate and bind cloth fibre. To apply the dye, the dye industry reductively converts the insoluble indigo to the water soluble leuco-indigo form via two-electron reduction in a batch process. The leuco-indigo is then oxidised by atmospheric oxygen to return the insoluble indigo, which adsorbs to cloth fibre. Unlike other synthetic blue dyes, indigo adsorbs to the cloth fibre instead of forming covalent bonds. The adsorbed indigo is robust to harsh detergents used for laundering, yet it wears out with persistence abrasion to reveal the white fibre core, which results in a desired worn-out look for a personalised jean and renders it irreplaceable by other synthetic blue dyes. ${ }^{[33]}$

Bond et.al reported the electrochemistry of solid state indigo by "mechanically attachment" to a basal plane pyrolytic graphite electrode (BPPG), pH buffered at a range of 5.0 to $13.0 .{ }^{[27 b]}$ In our work, a series of cyclic voltammograms were recorded using an indigo carbon/PVDF composite buffered at the corresponding $\mathrm{pH}$ values, shown in Figure 7. The voltammograms start at $-0.4 \mathrm{~V}$ and were swept negatively at a scan rate of $10 \mathrm{mVs}^{-1}$ to $-2.0 \mathrm{~V}$ vs MSE. A large reductive wave was seen at $-1.5 \mathrm{~V}$ vs MSE, labelled process 1 . On the reverse scan, two reversible redox processes, labelled process 2 and 3 are seen with formal potentials of $-0.72 \mathrm{~V}$ and $+0.07 \mathrm{~V}$ vs MSE at $\mathrm{pH} 5.0$ respectively. Process 1 was only seen on the first cycle and ascribed as the "break-in" reaction. ${ }^{[27 b]}$ Both of the midpoint potentials for process 2 and 3 were found to vary with pH by $-57 \pm 3.0 \mathrm{mV}$ and $-63.4 \pm 1.4 \mathrm{mV}$ per pH 
unit respectively, in agreement with the results reported by Bond et.al. ${ }^{[27 b]}$ The voltammograms obtained using the carbon/PVDF composite was mostly identical to that mechanically attached to BPPG, except that additional waves was seen at $\mathrm{pH} 9.0$ and 13.0. At a higher scan rate of $30 \mathrm{mVs}^{-1}$, multiple peaks appear with each process and have no correlation to the buffered $\mathrm{pH}$. This phenomenon was ascribed to the poor $\mathrm{pH}$ buffering inside the composite. The diffusion of buffering species into the composite is slow, which is analogous to the observation of back wave for the oxidation of OBD. The slow buffering effect is similar to that observed with a partially buffered solution, where the $\mathrm{pH}$ at the electrochemical interface alters as the finite $\mathrm{pH}$ buffering species were consumed. ${ }^{[19 b]}$ Therefore, caution is needed in interpreting voltammograms obtained with the carbon/PVDF composite when the electron transfer is accompany by the uptake of protons or hydroxide ions.
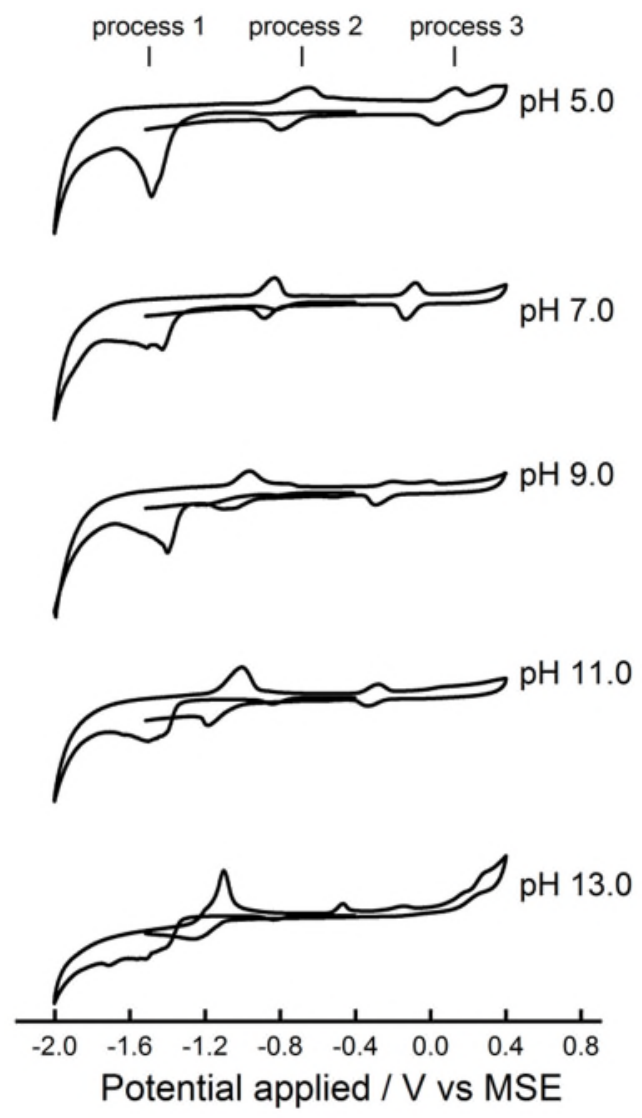

Figure 7. Cyclic voltammograms recorded on indigo carbon/PVDF composite modified electrodes immersed in degassed $0.1 \mathrm{M} \mathrm{KNO}_{3}$ and $\mathrm{pH}$ buffered solution. The potential was first swept negatively from $-0.40 \mathrm{~V}$ to $-2.00 \mathrm{~V}$, then reverse direction and swept positively to +0.40 $\checkmark$ before returning to $-1.50 \mathrm{~V}$ vs MSE. The composite contains a mixture of indigo, carbon black and PVDF in weight ratio of 20:60:20.

In summary, the carbon/PVDF composite electrode has demonstrated its ability to facilitate the electrochemistry of various solid particles. By imbedding the solid materials in the layers of cabon/PVDF, the diffusion of the formed charged species into bulk solution is hindered and 
information on the product can be obtained during the return sweep. It can however, lead to a complicated voltammogram in $\mathrm{pH}$ buffered solutions with electrochemical processes that involves the uptake of protons or hydroxide ions. This is due to the hinder diffusion of species from the bulk solution into the carbon/PVDF composite, which results a 'slow' buffering effect. Moreover, atmospheric oxygen was found adsorbed in the composite, the signals can be distinguished from the solid of interest by varying the weight ratios of the solid or to fabricate the carbon/PVDF electrode under nitrogen atmosphere.

Having understood the electrochemical behaviours of the carbon/PVDF composite the next section turns to focus the redox chemistry of $\alpha-\mathrm{HgS}$.

\section{$\alpha-H g S$}

The electrochemical analyses of solid $\alpha-\mathrm{HgS}$ were facilitated by using $\alpha-\mathrm{HgS}$ carbon/PVDF composite modified GC electrodes constructed as described above. The $\alpha$-HgS-carbon-PVDF composite layer is typically $100-1000 \mu \mathrm{m}$ in thickness. The inlay a) and b) of Figure 8 shows the surface topography imaged with a scanning electron microscope using backscattering (BSE) and secondary electron (SE) detectors respectively. The secondary electron image shows presence of solid $\alpha$-HgS particles, of sub-microns in size, on the surface of the carbon/PVDF composite. In contrast to the backscattering electron images, it reveals that the majority of the solid $\alpha-\mathrm{HgS}$ are imbedded in the carbon/PVDF composite.

Figure 9 shows a series of cyclic voltammograms recorded on $\alpha$-HgS carbon/PVDF composite electrodes, with reverse potentials ranging from $-1.60 \mathrm{~V}$ to $-2.00 \mathrm{~V}$ vs MSE. On the forward sweep, no apparent peak was seen for the reduction of $\alpha$-HgS solid. On the backward sweep however, two oxidative signals were seen at potential of $-0.49 \mathrm{~V}$ and $+0.24 \mathrm{~V}$ vs MSE, and the magnitude of those peaks increases with a higher negative return potential. At these high negative potential, the solid $\alpha-$ $\mathrm{HgS}$ reduces together with the occurrence of hydrogen evolution reactions (HER) at the electrode interface. The reduction of solid $\alpha-\mathrm{HgS}$ likely forms metallic mercury and $\mathrm{S}^{2-}$, but the reduction peak was not seen for $\alpha$-HgS as the current was dominated by the HER. Since the standard reduction potential of $\mathrm{Hg}^{2+} / \mathrm{Hg}$ is $+0.85 \mathrm{~V}$ vs SHE $\left(+0.21\right.$ vs MSE), ${ }^{[34]}$ the oxidative peak at $+0.24 \mathrm{~V}$ vs MSE is ascribed to the anodic stripping of metallic mercury and $-0.49 \mathrm{~V}$ vs MSE is the oxidation of sulphides within the composite. The inlay b) in Figure 9 shows a plot of the mercury stripping charge verses return potential and sharp gradient can be seen around -1.8 to $-1.9 \mathrm{~V}$. The stripping charge of mercury will be directly correlated to the amount $\alpha-\mathrm{HgS}$ reduced; the reduction peak potential of $\alpha$ $\mathrm{HgS}$ is therefore within the range of -1.8 to $-1.9 \mathrm{~V}$ vs MSE. Furthermore, inlay a) in Figure 9 shows a 
square wave voltammogram measured on the $\alpha$-HgS carbon/PVDF composite electrode with a reductive peak appearing at a potential of $-1.82 \mathrm{~V}$ vs MSE, which is not dissimilar to the differential wave voltammogram reported by Scholz et.al using a paraffin impregnated graphite electrode. ${ }^{[15]}$ Moreover, a number of reductive spikes were seen after the return potential of $-1.95 \mathrm{~V}$ and $-2.00 \mathrm{~V}$ vs MSE; this can be tentatively ascribed to the reduction of large HgS particles as a result of an applied over-potential.

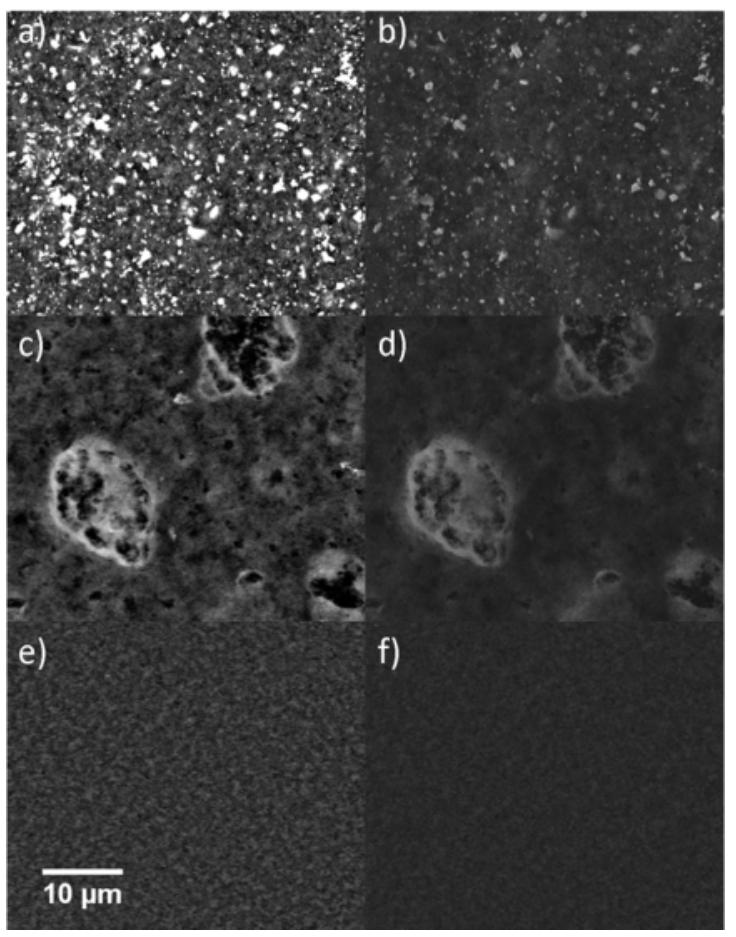

Figure 8. Scanning electron microscope images of various carbon/PVDF composites using the back scatting electron detector (a, c and e) and secondary electron detector $(b, d$ and $f)$. The brightness and contrast settings were the same for backscattering electron images. The $\alpha$ $\mathrm{HgS}$ carbon/PVDF composite - inlay a) and b); sulphur carbon/PVDF composite - inlay c) and d); the weight ratio of the composite is 20:60:20 ( $\alpha-\mathrm{HgS} / \mathrm{S}$ : carbon black : PVDF). Blank carbon/PVDF composite - inlay e) and f), the weight ratio of carbon black to PVDF is 60:20.

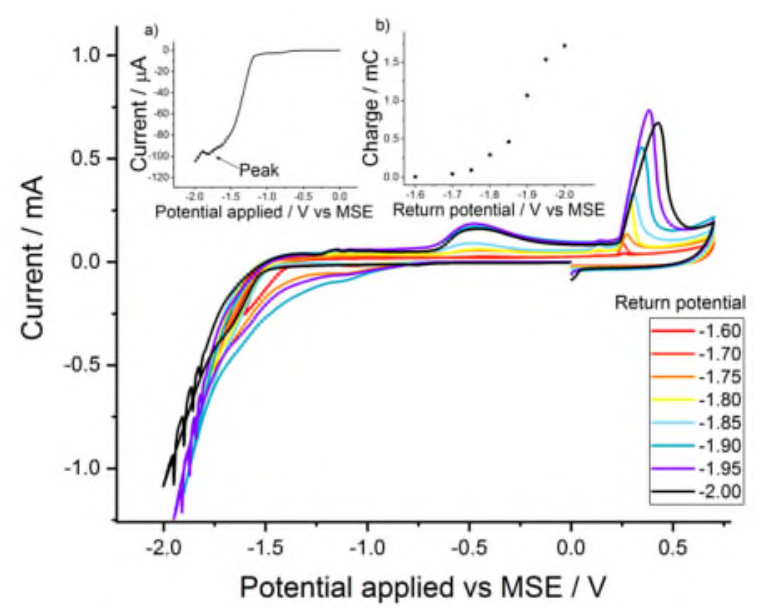

Figure 9. Cyclic voltammograms of glassy carbon electrode modified by dropcasting a composite of $\alpha$-HgS, carbon black and PVDF, recorded in degassed $\mathrm{pH}$ buffered solution of $0.10 \mathrm{M} \mathrm{KNO}_{3}$ at a scan rate of $10 \mathrm{mVs}^{-1}$. Inlay, a) reductive square wave voltammogram of the 
$\alpha$-HgS composite modified GC electrode with experimental parameters of $2 \mathrm{mV}$ step potential, modulation amplitude of $10 \mathrm{mV}$ and frequency of $10 \mathrm{~Hz}$. Inlay, b) plot of charge against the return potential for the oxidative peak at +0.24V vs MSE in Figure 9.

Figure 10 shows a voltammogram measured with a blank carbon/PVDF composite electrode immersed in a $1.0 \mathrm{mM} \mathrm{Hg}^{2+}$ solution. The SEM images of the blank carbon/PVDF composite are shown in the inlay e) and f) of Figure 8. The voltammogram swept positively from $-1.80 \mathrm{~V}$ and an oxidative signal was seen at $+0.15 \mathrm{~V}$ vs MSE. During the forward sweep, mercury deposits on the blank carbon/PVDF composite via two-electron reduction of $\mathrm{Hg}^{2+}$, the oxidation signal at $+0.15 \mathrm{~V}$ vs MSE is therefore the anodic stripping of metallic mercury to reform $\mathrm{Hg}^{2+}$. Interestingly, the stripping potential of mercury recorded on a blank carbon/PVDF composite, $+0.15 \mathrm{~V}$, is slight lower than that observed with the $\alpha-\mathrm{HgS}$ carbon/PVDF composite, $+0.24 \mathrm{~V}$. This discrepancy can be ascribed to the different in local chemical environment of the mercury. On the oxidative sweep after the reduction of $\alpha-\mathrm{HgS}$ composite, sulphides were presumed to oxidise to form sulphur prior to the anodic stripping of mercury. Possibly it is an interaction ${ }^{[35]}$ between sulphur and mercury that causes a slightly higher mercury stripping potential in comparison to that seen for no presence of sulphur. Furthermore, small amount of sulphur was added into the carbon/PVDF composite to form a sulphur-carbon-PVDF composite with a weight ratio of 3:60:20. The inlay c) and e) of Figure 8 shows the backscattering and secondary electron SEM images of the sulphur-carbon-PVDF composite. The cyclic voltammogram measured with this sulphur-carbon-PVDF composite immersed in a $1.0 \mathrm{mM}$ $\mathrm{Hg}^{2+}$ solution is shown in Figure 11. Two anodic mercury stripping signals can be seen at a potential of $+0.11 \mathrm{~V}$ and $+0.22 \mathrm{~V}$ vs MSE. It is evident that, in the limiting sulphur environment, the first oxidation peak at $+0.11 \mathrm{~V}$ is the stripping of mercury in a chemical environment without sulphur and the second at $+0.22 \mathrm{~V}$ is that in presence of sulphur environment. Thus it can be concluded that, the anodic stripping potential of mercury shifts positively in presence of a local sulphur-rich environment.

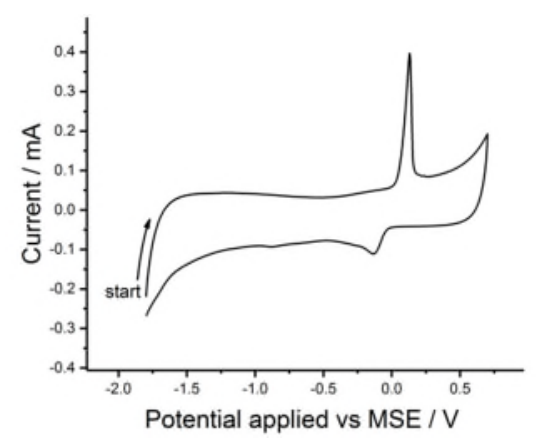

Figure 10. Second cycle of the cyclic voltammogram measured with a carbon/PVDF composite modified GC electrode, at a scan rate of 50 $\mathrm{mVs}^{-1}$ in degassed aqueous solution containing $1.0 \mathrm{mM} \mathrm{HgNO}_{3}$ and 0.10 $\mathrm{M} \mathrm{KNO}_{3}$. The weight ratio of carbon black to PVDF is 60:20. 


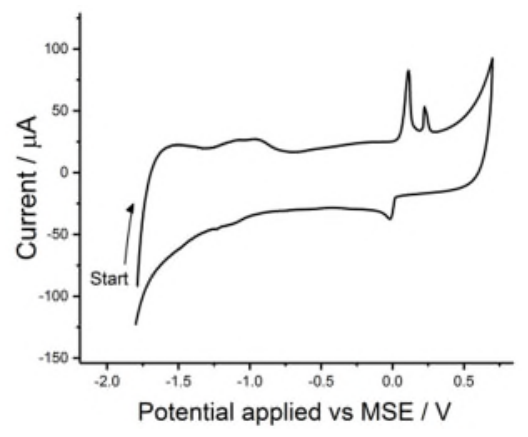

Figure 11. Second cycle of the cyclic voltammogram measured with a sulphur carbon/PVDF composite modified GC electrode, at a scan

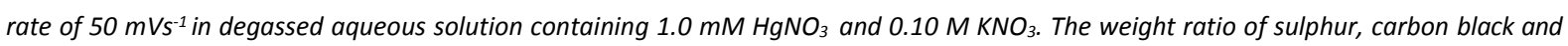
PVDF in the composite is 3:60:20.

Figure 12 shows a series of voltammograms recorded on carbon/PVDF composites containing different ratios of sulphur and $\alpha$-HgS. The oxidation peak potential of sulphide in the absence of $\alpha$ $\mathrm{HgS}$ occurs at $-0.75 \mathrm{~V}$ vs MSE. As the ratio of sulphur to mercury sulphide decreases, the oxidation peak potential of sulphide ions shifts positively, to a potential of $-0.49 \mathrm{~V}$ vs MSE with carbon/PVDF composite containing only $\mathrm{HgS}$. The shift in the sulphide oxidation potential is analogous to that seen with mercury described above, i.e. the oxidation peak potential of sulphide ions shift positively in the presence of a local mercury-rich environment and vice versa.

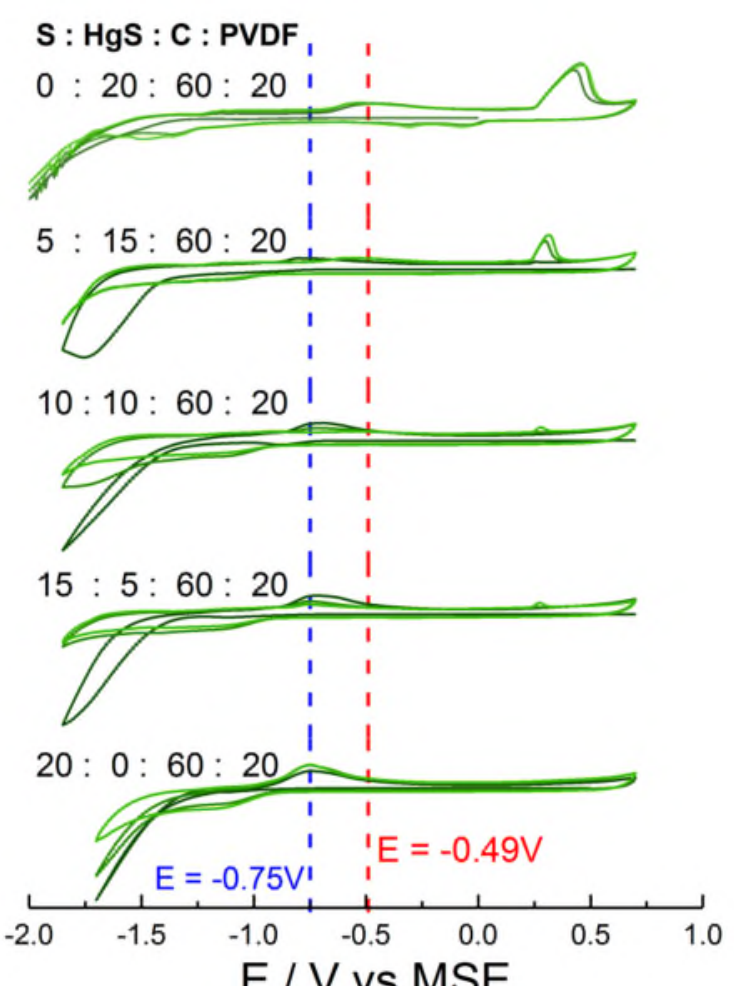

Figure 12. Cyclic voltammograms of carbon/PVDF composite containing different ratio of HgS and S, 3 consecutive cycles were recorded in degassed $0.1 \mathrm{M} \mathrm{KNO}_{3}$. The cycles 1 to 3 were displayed in colours from dark to light green respectively. 


\section{Conclusions}

In this article we have presented a complementary method facilitating the electrochemical study of insoluble particulate compounds. By modifying a glassy carbon electrode with a composite of $\alpha-\mathrm{HgS}$, carbon black and PVDF, the cyclic voltammogram of $\alpha-\mathrm{HgS}$ is shown and characterised for the first time. Using this carbon/PVDF composite, the reduction peak potential of $\alpha-\mathrm{HgS}$ was found in the square wave voltammogram at $-1.82 \mathrm{~V}$ vs MSE and the subsequent oxidation of sulphide and metallic mercury occurred at potentials of $-0.49 \mathrm{~V}$ and $+0.24 \mathrm{~V}$ vs MSE respectively, both shifted slightly positively compare to that measured in isolation using a blank carbon/PVDF composite. This shift in potential is ascribed to the interaction of mercury with the local sulphide environment, and vice versa.

The use of this carbon/PVDF composite offers several advantages over other solid state electrochemical methods. In comparison to mechanical transfer methods, the carbon/PVDF composite utilises dropcasting to accurately control the amount of reagents; comparative studies between experiments can therefore be made. Since the reactant is mostly imbedded in the carbon/PVDF composite, the diffusion of the formed products into solution is hindered and a back signal can be observed which otherwise might be lost with surface adsorption or abrasive methods. However, for electrochemical processes that involve the uptake of protons or hydroxide ions, the layers of carbon/PVDF can hinder the diffusion of species from bulk solution into the composite and thus may result in a slow buffering effect; the experimental conditions can be optimised to reduce this effect, by lowering the scan rate and reducing the dropcast volume for a thinner layer of carbon/PVDF composite - this allows longer time for the solution phase species to diffuse and across a shorter distance into the composite. A small reduction signal at $-0.97 \mathrm{~V}$ vs MSE was attributed to the adsorption of atmospheric oxygen in the carbon/PVDF composite; this was insignificant in our voltammetric studies but it is possible to conduct the fabrication process and experiment under nitrogen environment if desired.

\section{Supporting Information}

The reproducibility the carbon composite electrode are shown in in Supporting Information, where repeated cyclic voltammetry experiments of $\mathrm{HgS}$ carbon composite resulted mercury stripping charge of $1.00 \pm 0.14 \mathrm{mC}$ (return potential at $-1.9 \mathrm{~V}$ vs MSE).

\section{Acknowledgements}


The research leading to these results has received partial funding via an EPSRC Industrial CASE award and the European Research Council under the European Union's Seventh Framework Programme (FP/2007-2013) / ERC Grant Agreement n. [320403].

\section{References}

[1] a) W. I. Mercury, Geneva: World Health Organization 1991, 107; b) M. Berlin, R. Zalups, B. Fowler, Handbook on Toxicology of Metals 1986; c) R. A. Bernhoft, Journal of Environmental and Public health 2012, 2012.

[2] Methylmercury, World Health Organization 1990.

[3] a) M. Harada, Critical Reviews in Toxicology 1995, 25, 1-24; b) M. Harada, Teratology 1978, 18, 285-288.

[4] a) L. Yuan-Fu, W. Qin, Y. Jun-Wen, S. Jing-Zhen, L. Jie, S. Jing-Shan, Experimental Biology and Medicine 2011, 236, 233-239; b) J. Liu, J.-Z. Shi, L.-M. Yu, R. A. Goyer, M. P. Waalkes, Experimental Biology and Medicine 2008, 233, 810-817.

[5] a) J. R. Craig, P. B. Barton, Economic Geology 1973, 68, 493-506; b) R. Potter, H. Barnes, American Mineralogist 1978, 63, 1143-1152; c) H. L. Clever, S. A. Johnson, M. E. Derrick, Journal of Physical and Chemical Reference Data 1985, 14, 631-680.

[6] a) F. Dickson, G. Tunell, American Mineralogist 1959, 44, 471; b) E. T. Allen, J. L. Crenshaw, H. E. Merwin, American Journal of Science 1912, 341-396; c) C. V. King, Annals of the New York Academy of Sciences 1957, 65, 360-368.

[7] G. Kullerud, Carnegie Inst. Washington 1965, Yearbook 64b, 193.

[8] a) L. G. Hepler, G. Olofsson, Chemical Reviews 1975, 75, 585-602; b) W. Treadwell, F. Schaufelberger, Helv. Chim. Acta 1946, 29, 1936-1946; c) A. Ringbom, Analytical Section, International Union of Pure and Applied Chemistry 1953; d) H. C. Helgeson, American Journal of Science 1969, 267, 729-804; e) R. A. Robie, B. S. Hemingway, US Geol. Survey Bull., vol. 2131, p. 461-461 (1995). 1995, 2131, 461-461; f) J. R. Goates, A. G. Cole, E. L. Gray, J. Am. Chem. Soc. 1951, 73, 3596-3597; g) J. R. Goates, M. B. Gordon, N. D. Faux, J. Am. Chem. Soc. 1952, 74, 835-836; h) A. Ringbom, Report to AnaL Sect. IUPAC 1953.

[9] a) A. J. Bard, L. R. Faulkner, Electrochemical Methods: Fundamentals and Applications, Wiley, 2000; b) R. Compton, C. Banks, Imperial College Press, 2011.

[10] F. Scholz, B. Lange, TrAC Trends in Analytical Chemistry 1992, 11, 359-367.

[11] R. N. Adams, Anal. Chem. 1958, 30, 1576-1576.

[12] a) Z. Rozhdestvenskaya, I. K. Muldagalieva, A. Zharmenov, Vol. 18, Soviet Electrochemistry, 1982, pp. 111-114; b) K. Z. Brainina, Talanta 1987, 34, 41-50; c) V. Barikov, R. ZB, O. Songina, Industrial laboratory 1969, 35, 928; d) Z. Rozhdestvenskaya, V. Sigitov, O. Songina, Journal of Analytical Chemistry of the USSR 1979, 34, 350-353; e) O. Songina, I. Trushina, Z. B. Rozhdestvenskaya, N. Cherkasova, Ehlektrokhimiya 1974, 9, 1310-1312.

[13] a) F. Scholz, B. Meyer, Chemical Society Reviews 1994, 23, 341-347; b) F. Scholz, Journal of Solid State Electrochemistry 2013, 17, 1493-1504; c) F. Scholz, N. A. Kolpakova, J. D. Sister, V. G. Mairanovsky, in Electrochemistry in a Divided World, Springer, 2015, pp. 97-178; d) A. Doménech-Carbó, J. Labuda, F. Scholz, Pure and Applied Chemistry 2012, 85, 609-631; e) F. Scholz, U. Schröder, R. Gulaboski, A. Doménech-Carbó, in Electrochemistry of Immobilized Particles and Droplets, Springer, 2015, pp. 1-10.

[14] a) I. Švancara, K. Vytřas, K. Kalcher, A. Walcarius, J. Wang, Electroanalysis 2009, 21, 7-28; b) I. Švancara, A. Walcarius, K. Kalcher, K. Vytřas, Central European Journal of Chemistry 2009, 7, 598-656; c) K. Vytřas, I. Švancara, R. Metelka, Journal of the Serbian Chemical Society 2009, 
74, 1021-1033; d) T. Mikysek, M. Stoces, I. Svancara, K. Vytras, Chemicke Listy 2014, 108, 513-518.

[15] A. M. Bond, F. Scholz, Langmuir 1991, 7, 3197-3204.

[16] a) A. Doménech-Carbó, M. T. Doménech-Carbó, M. Moya-Moreno, J. V. Gimeno-Adelantado, F. Bosch-Reig, Analytica Chimica Acta 2000, 407, 275-289; b) A. Doménech - Carbó, M. Dom énech - Carbó, L. Osete - Cortina, Electroanalysis: An International Journal Devoted to Fundamental and Practical Aspects of Electroanalysis 2001, 13, 927-935; c) A. Doménech, H. García, I. Casades, M. Esplá, The Journal of Physical Chemistry B 2004, 108, 20064-20075; d) A. Doménech, H. García, M. Alvaro, E. Carbonell, The Journal of Physical Chemistry B 2003, 107, 3040-3050.

[17] D. Lowinsohn, P. Gan, K. Tschulik, J. S. Foord, R. G. Compton, Electroanalysis 2013, 25, 24352444.

[18] C. Batchelor-McAuley, M. Yang, E. M. Hall, R. G. Compton, J. Electroanal. Chem. 2015, 758, 16.

[19] a) P. S. Guin, S. Das, P. C. Mandal, International Journal of Electrochemistry 2011, 2011, 22; b) M. Yang, C. Batchelor-McAuley, E. Kätelhön, R. G. Compton, Anal. Chem. 2017, 89, 68706877.

[20] a) M. Shamsipur, A. Siroueinejad, B. Hemmateenejad, A. Abbaspour, H. Sharghi, K. Alizadeh, S. Arshadi, J. Electroanal. Chem. 2007, 600, 345-358; b) C. Rüssel, W. Janicke, J. Electroanal. Chem. Interfacial Electrochem. 1986, 199, 139-151.

[21] C. Batchelor-McAuley, B. R. Kozub, D. Menshykau, R. G. Compton, J. Phys. Chem. C 2011, 115, 714-718.

[22] J. Jacq, J. Electroanal. Chem. Interfacial Electrochem. 1971, 29, 149-180.

[23] M. Yang, C. Batchelor-McAuley, E. Kätelhön, R. G. Compton, Anal. Chem. 2017, 89, 68706877.

[24] C. Batchelor-McAuley, Q. Li, S. M. Dapin, R. G. Compton, J. Phys. Chem. B 2010, 114, 40944100.

[25] a) M. E. Tessensohn, H. Hirao, R. D. Webster, J. Phys. Chem. C 2013, 117, 1081-1090; b) P. S. Guin, S. Das, P. Mandal, Int. J. Electrochem. Sci 2008, 3, 1016-1028; c) N. Gupta, H. Linschitz, J. Am. Chem. Soc. 1997, 119, 6384-6391.

[26] T. Kuwana, W. French, Anal. Chem. 1964, 36, 241-242.

[27] a) A. M. Bond, J. B. Cooper, F. Marken, D. M. Way, J. Electroanal. Chem. 1995, 396, 407-418;

b) A. M. Bond, F. Marken, E. Hill, R. G. Compton, H. Hugel, Journal of the Chemical Society, Perkin Transactions 2 1997, 1735-1742.

[28] R. G. Compton, C. E. Banks, Understanding Voltammetry, World Scientific, 2007.

[29] X.-F. Zhou, W. Cheng, R. G. Compton, Nanoscale 2014, 6, 6873-6878.

[30] a) B. Zinger, J. Electroanal. Chem. Interfacial Electrochem. 1988, 239, 209-225; b) M. A. G. Trindade, M. V. B. Zanoni, Sensors and Actuators B: Chemical 2009, 138, 257-263.

[31] R. Peters, The Physical Chemistry of Dyeing 1975.

[32] a) L. Wolf, Chem. Eng. News 2011, 89, 44; b) C. B. Schimper, C. Ibanescu, T. Bechtold, Biotechnology Journal 2011, 6, 1280-1285.

[33] T. M. Hsu, D. H. Welner, Z. N. Russ, B. Cervantes, R. L. Prathuri, P. D. Adams, J. E. Dueber, Nature chemical biology 2018.

[34] A. F. Cotton, G. Wilkinson, M. Bochmann, C. A. Murillo, Advanced Inorganic Chemistry, Wiley, 1999.

[35] a) R. G. Pearson, Journal of Chemical Education 1968, 45, 581; b) R. G. Pearson, Journal of Chemical Education 1968, 45, 643. 